\title{
Performance evaluation of an all-fiber image-reject homodyne coherent Doppler wind lidar
}

\author{
C. F. Abari, A. T. Pedersen, E. Dellwik, and J. Mann \\ Department of Wind Energy, Technical University of Denmark (DTU), Roskilde 4000, Denmark \\ Correspondence to: C. F. Abari (cyrus.abari@gmail.com)
}

Received: 27 January 2015 - Published in Atmos. Meas. Tech. Discuss.: 14 April 2015

Revised: 23 September 2015 - Accepted: 24 September 2015 - Published: 9 October 2015

\begin{abstract}
The main purpose of this study is to evaluate the near-zero wind velocity measurement performance of two separate $1.5 \mu \mathrm{m}$ all-fiber coherent Doppler lidars (CDLs). The performance characterization is carried out through the presentation of the results from two separate atmospheric field campaigns. In one campaign, a recently developed continuous wave $(\mathrm{CW}) \mathrm{CDL}$ benefiting from an image-reject front-end was deployed. The other campaign utilized a different CW CDL, benefiting from a heterodyne receiver with intermediate-frequency (IF) sampling. In both field campaigns the results are compared against a sonic anemometer, as the reference instrument. The measurements clearly show that the image-reject architecture results in more accurate measurements of radial wind velocities close to zero. Close-to-zero velocities are usually associated with the vertical component of the wind and are important to characterize.
\end{abstract}

\section{Introduction}

Light detection and ranging (lidar) for remote sensing of wind has become a well-established and widely used instrument in atmospheric science and wind energy (Mayor and Eloranta, 2001; Wang et al., 2010; Grund et al., 2001; Schlipf et al., 2013; Smith et al., 2006; Bingöl et al., 2010; Pichugina et al., 2012). Among different variants of lidars, coherent Doppler lidars (CDLs) are of primary interest for remote measurement of wind as well as characterization of turbulence structures for the lower atmosphere (Menzies and Hardesty, 1989; Hall et al., 1984; Sathe and Mann, 2013). Due to their nature of operation, CDLs measure the radial velocity of the wind which does not necessarily coincide with the true velocity vector. Thus, one ideally needs to employ three lidars, with a sufficient angular separation, for probing the measurement volumes of interest to be able to derive the full wind velocity vector. One of the challenges in existing CDLs is the detection of the radial velocity direction. Among the few commercially available continuous wave (CW) systems none is capable of determining the radial velocity direction.

A few research CW CDLs, capable of determining the sign of the radial velocity, have been developed over the years. For instance, Schwiesow and Cupp (1981) used two $\mathrm{CO}_{2}$ lasers with frequency-offset locking to discriminate the radial velocity direction. The system benefits from a down-conversion principle known as heterodyne receiver with intermediate-frequency (IF) sampling (Shimizu et al., 1994; Razavi, 1997). However, the reported signal-to-noise ratio around zero velocity in this system was poor. More recently, a CW CDL capable of determining the radial velocity sign/direction is the first-generation "Windscanner" (Mikkelsen et al., 2014) also benefiting from a heterodyne receiver with IF sampling. In this system an acousto-optic modulator (AOM) is used to provide a frequency shift (offset) between the local oscillator (LO) signal and the transmit signal. As an all-fiber directional CW CDL, the firstgeneration Windscanner has been a valuable research instrument for directional remote sensing of wind. A detailed analysis of systems benefiting from the heterodyne front-ends with IF sampling is beyond the scope of this paper. It suffices to mention that they may suffer from a number of drawbacks in terms of (more) extraneous noise, (lower) detection bandwidth (BW), as well as (more) intensive data acquisition and processing. Some of these issues are briefly discussed in the remainder of this paper. 
Recently, an all-fiber directional CW CDL employing an image-reject homodyne optical front-end was successfully demonstrated by Abari et al. (2014). This reported system utilizes an all-fiber $90^{\circ}$ hybrid (Kylia), conventionally employed in high-speed optical communications, to optically down-convert the desired signals to baseband. As opposed to the heterodyne receivers with IF sampling, the optical down conversion is carried out with passive components only. As a result, the noise behavior of the system, especially around the zero Doppler shift, is improved. Besides, the system reduces the $\mathrm{BW}$ of the photo-detectors as well as the analog-to-digital converter by a factor of 2. Abari et al. (2014) have shown that, due to the presence of two signal components with independent noise sources, a cross-spectral analysis technique can be utilized to remove the unnecessary noise sources in the system, eliminating the additional intensive signal processing for the removal of the background noise.

To evaluate the performance of the all-fiber image-reject system (see Abari et al., 2014), its performance was compared against a sonic anemometer in a field campaign. The measurements were specifically carried out to measure the vertical component of the wind vector: the vertical component is usually very small and appears in the frequency region where CW CDLs generally suffer from a multitude of noise sources, such as offset noise, interferometric noise, $1 / f$ noise, etc. For comparison purposes, the results of this campaign are compared with the results of a different campaign carried out in 2013 where three first-generation Windscanners (benefiting from an AOM-based heterodyne receiver with IF sampling) were utilized to measure the 3-D wind vector. For the latter, only the results associated with measured radial velocities close to zero are discussed in this paper so that a fair comparison between the above-mentioned systems can be made.

This paper starts with a brief and simple introduction, in terms of baseband signal models, to the image-reject architecture and how it compares to the heterodyne architecture with IF sampling. We also discuss the advantages and disadvantages of a signal processing approach, introduced in Abari et al. (2014) and further analyzed in Pedersen et al. (2014), to remove the dominant noise sources and eliminate spectral whitening. Then, we will present some of the measurement results relevant to this paper for two separate measurement campaigns where the first-generation Windscanners and a prototype CW CDL, benefiting from image-reject homodyne receiver (Abari et al., 2014), were deployed for remote sensing of wind.

Finally, the paper is wrapped up with a few concluding remarks. Throughout this paper, we will use ICDL and HCDL to refer to the CW CDL benefiting from image-reject frontend and first-generation Windscanner CW CDLs (an AOMbased heterodyne receiver with IF sampling), respectively.

\section{Image-reject optical receiver in CW CDLs and spectral processing}

One of the most well-known and widely used optical frontend architectures in CW CDLs is the homodyne receiver with real mixing (Karlsson et al., 2000). A detailed analysis of this system, as well as other architectures, is not the purpose of this paper. The interested reader can refer to Abari et al. (2014) and Karlsson et al. (2000) for more information. In such a system, a simplified transmit signal can be expressed as

$s(t) \propto \cos \left(2 \pi f_{\mathrm{c}} t\right)$,

where $f_{\mathrm{c}}$ is the optical carrier frequency. As a result, the baseband signal associated with backscatter from a single moving particle can be written as

$i(t)=\alpha \cos (2 \pi \Delta f t)$,

where $\alpha$, among other things, represents the net effect of transmit optical power, atmospheric transmission, scattering, telescope area, and the receiver efficiency. In Eq. (2) we have ignored any parameters (such as phase shift) secondary to the concepts discussed in this paper. Please note that Eq. (2) represents both negative and positive Doppler shifts. As a result, due to its symmetric spectrum with respect to the zero frequency it is impossible to infer the direction of the radial velocity.

Figure 1 illustrates an example of a HCDL where the role of the acousto-optic modulator (AOM) is to shift the LO frequency to an IF offset to enable the discrimination of negative and positive radial velocities. ${ }^{1}$ Assuming similar operating conditions, the detected signal, in the baseband form, for the transmit signal in Eq. (1) is

$i(t)=\alpha \cos \left(2 \pi f_{\mathrm{IF}} t \pm 2 \pi \Delta f t\right)$.

As we can see from Eq. (3), it is relatively simple to extract the sign of the radial velocity as well as its magnitude; the sign can be inferred by comparing the Doppler shift with respect to the IF. However, we know from experience that a few imperfections contribute to the corruption of the desired Doppler signal components close to IF. We believe the main sources of spurious signals are leakage from the optical circulator, back reflections from the telescope ${ }^{2}$ and a challenging offset noise removal at the IF. Besides, possible AOM imperfections, such as a dirty AOM radio frequency drive and

\footnotetext{
${ }^{1}$ Alternatively, the transmit (or receive) signal can be frequency shifted. In first-generation Windscanners, the transmit signal is frequency shifted.

${ }^{2}$ In CW CDLs, the presence of non-ideal characteristics of the optical circulator and anti-reflection coating may give rise to an unwanted signal in the vicinity of the zero-velocity Doppler component. The unwanted signal is known as the interferometric noise. Moslehi (1986) can be consulted for a thorough analysis of interferometric noise in coherent fiber-optic systems.
} 


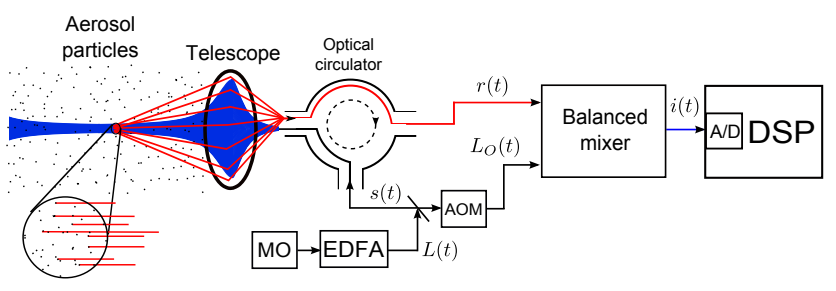

Figure 1. Heterodyne receiver with IF sampling (HCDL). To be able to capture the full return signal power a balanced mixer/detector needs to be employed; for details please see Abari et al. (2014). MO and EDFA represent the master oscillator and erbium-doped fiber amplifier, respectively. Optical circulator isolates the transmit, $s(t)$, and the receive signal, $r(t)$. $L_{\mathrm{O}}(t)$ represents the local oscillator signal, A/D is the analog-to-digital converter, and DSP is the digital signal processor unit.

the zeroth-order component leakage, may contribute to additional noise in the system. As a result, an accurate measurement of small Doppler shifts (associated with wind speeds close to zero) becomes more cumbersome and sometimes even impossible.

A thorough analysis of an all-fiber image-reject homodyne receiver has been provided in Abari et al. (2014). This system utilized a receiver employing two signal detection arms: inphase (I) and quadrature-phase (Q) components. The combination of the I and Q signal components results in a complexvalued signal

$i(t)=i_{\mathrm{I}}(t)+j i_{\mathrm{Q}}(t)=\frac{\alpha \sqrt{2}}{2} \cos (2 \pi \Delta f t) \pm j \frac{\alpha \sqrt{2}}{2} \sin (2 \pi \Delta f t)$,

where $j=\sqrt{-1}$. Furthermore, $i_{\mathrm{I}}(t)$ and $i_{\mathrm{Q}}(t)$ are the baseband I and Q components, respectively. It can readily be seen that by comparing the I and Q components in Eq. (4), the radial velocity sign can be inferred. Furthermore, we have shown in Abari et al. (2014) that there are two approaches to retrieve the velocity component from the spectral analysis of Eq. (4), i.e., auto-spectral analysis of the complex signal or the cross-spectral analysis of the in-phase and quadrature components. For the remainder of this paper, we use the term cross-spectral approach when referring to the cross-spectrum between the in-phase and quadrature components of the baseband signal in Eq. (4). The cross-spectral approach seems to be the obvious option in the majority of measurements due to its ability to remove, at least on average, the uncorrelated noise sources such as the dominant detector's shot noise. The main advantage of this approach, for the majority of scenarios, is the elimination of additional signal processing algorithms, such as spectral whitening, that may introduce additional estimation noise. However, as will be shown shortly, the cross-spectral approach cannot be reliably employed for a small number of measurement cases using the ICDL where the Doppler spectra leak across the zero frequency.

Following Eqs. (25) and (26) in Abari et al. (2014) it is evident that the cross-spectral approach works best when

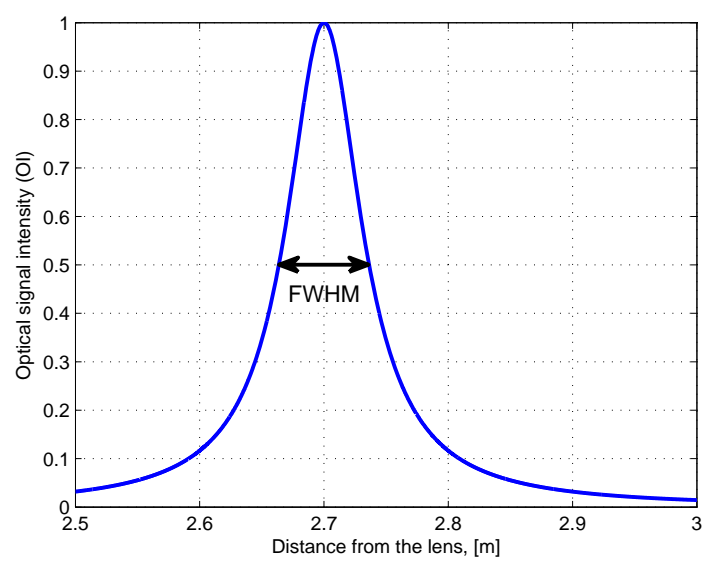

Figure 2. Optical signal intensity as a function of distance from the output lens of a telescope. For an effective aperture diameter of $2 \mathrm{~cm}$, the FWHM at a focus distance of $2.7 \mathrm{~m}$ is about $72 \mathrm{~mm}$. Due to beam truncation at the output lens in our system, the measured FWHM is $140 \mathrm{~mm}$.

the spectral components are to the one side of the zero frequency. In other words, the Doppler shifts associated with the backscatter are either all positive or negative: they do not leak across the zero frequency. This is, of course, the case for the majority of scenarios. However, as we will show in this paper, the estimation of Doppler shifts distributed around zero frequency for cross-spectral approach becomes skewed and biased. For instance, if the vertical velocity component measurement associated with a large sampling volume is carried out, it is highly probable to observe a wide distribution of velocities which cross the zero frequency. This is indirectly due to the incapability of lidars to provide point measurements; CDLs always provide a volume measurement. In the event of CW CDLs, the sampling volume is primarily a function of the output lens diameter and measurement range.

For an untruncated Gaussian beam, the transmit laser beam's optical intensity (OI) has a Lorentzian distribution defined by

$\mathrm{OI}=\frac{\Gamma}{\pi\left[(F-d)^{2}+\Gamma^{2}\right]}$,

where $\lambda$ is the wavelength; $d$ and $F$ are the distance and focus distance of the light with respect to the output lens of the telescope, respectively. Furthermore,

$\Gamma=\frac{4 \lambda F^{2}}{\pi D_{\mathrm{eff}}^{2}}$,

where $D_{\text {eff }}$ is the output lens effective diameter, i.e., where the transmit beam radial intensity drops to $1 / e^{2}$ (see Sonnenschein and Horrigan, 1971). For an effective antenna diameter of $2 \mathrm{~cm}$ and a focus distance of $2.7 \mathrm{~m}$ the full width at half maximum (FWHM) of the beam (as shown in Fig. 2) is $72 \mathrm{~mm}$ (see Sonnenschein and Horrigan, 1971; Angelou 
et al., 2012b). In our experiment the lens diameter (not the effective diameter) was a mere $D=2.2 \mathrm{~cm}$. Due to beam truncation (see Urey, 2004) at the output lens of the telescope in our system the FWHM at the focus distance deviated from the untruncated beam in Eq. (5). Our measurements indicated a FWHM of $140 \mathrm{~mm}$ at a focus distance of $2.7 \mathrm{~m}$ at the time of measurement. This width corresponds approximately to the $115 \mathrm{~mm}$ gap of the sonic anemometer (Horst and Oncley, 2006) used for the verification of the measurement results, elaborated in Sect. 3. Following Eq. (6) it can be inferred that the FWHM varies quadratically as a function of the focus distance.

To demonstrate the performance of the cross-spectral approach, in the event of Doppler spectral power at both sides of zero frequency, let us assume a simple case of optical backscatter from two individual aerosol particles. The two particles have Doppler shifts equal in magnitude but opposite in sign, with baseband coefficients $\alpha$ and $\beta$ associated with positive and negative Doppler shifts, respectively. Thus, assuming the transmit signal in Eq. (1) and following the image-reject architecture elaborated in Abari et al. (2014), the following baseband complex signal can be formulated

$$
\begin{aligned}
i(t) & =i_{\mathrm{I}}(t)+j i_{\mathrm{Q}}(t)=\frac{\sqrt{2}(\alpha+\beta)}{2} \cos (2 \pi \Delta f t) \\
& +j \frac{\sqrt{2}(\alpha-\beta)}{2} \sin (2 \pi \Delta f t) .
\end{aligned}
$$

Moreover, assuming the desired Doppler signal information is contained in the imaginary part of the cross-spectrum between I and Q Abari et al. (2014),

$\Im\left[P_{i_{1} i_{\mathrm{Q}}}\right]=\frac{(\alpha+\beta)(\alpha-\beta)}{8}[\delta(f+\Delta f)-\delta(f-\Delta f)]$,

where $\Im\left[P_{i_{\mathrm{I}} i_{\mathrm{Q}}}\right]$ represents the imaginary component of the cross-spectrum between I and Q.

To assess the performance of the cross-spectral approach, let us consider three different scenarios:

1. If $\beta \rightarrow 0$, then

$$
\Im\left[P_{i_{\mathrm{I}} i_{\mathrm{Q}}}\right]=\frac{\alpha^{2}}{8}[\delta(f+\Delta f)-\delta(f-\Delta f)],
$$

which is a better spectral estimator, compared to the auto-spectral method, as elaborated in Abari et al. (2014) and Pedersen et al. (2014). This is a very common measurement scenario since simultaneous occurrence of Doppler spectral components with opposite sign is rare and is expected in specific conditions, e.g., vertical wind component measurement in turbulent flow or large sampling volume.

2. If $\beta=\alpha$, then

$$
\mathfrak{\Im}\left[P_{i_{1} i_{Q}}\right]=0 .
$$

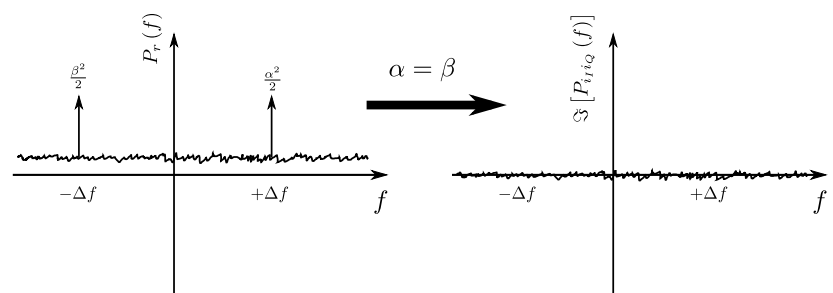

(a)

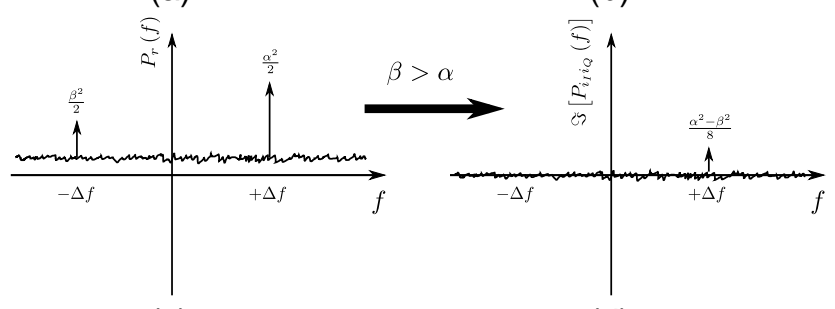

(c)

(d)

Figure 3. Cross-spectral approach in the event of spectral components appearing on both sides of zero frequency. Examples of autospectra are shown in the left column while the corresponding crossspectra are shown in the right.

In this case, contrary to the auto-spectral procedure, the estimator fails to detect the presence of a Doppler signal. However, the center of gravity and median estimators, explained in what follows, are able to produce the correct average Doppler shift associated with the sampling volume.

3. If $\beta \neq \alpha$ and $\beta \neq 0$, then Eq. (8) detects a single signal component which might be negative or positive depending whether $\beta>\alpha$ or $\beta<\alpha$. This may result in an inaccurate detection/estimation of the Doppler shift and introduce a bias in the measured volume-averaged velocity estimate away from zero.

As a result, although cross-spectral approach provides a reliable and convenient way for Doppler shift estimation in the majority of cases, it fails to provide unbiased velocity estimates when Doppler components spread across the zero frequency.

On the other hand, more often than not, we are interested in the mean value of the Doppler shift as it represents the dominant wind velocity in the sampling volume. Thus, is it possible to utilize the cross-spectral approach when one is interested in the average value of the wind velocity in the sampling volume? To answer this question, let us take the two practical estimators conventionally used for the samplingvolume average wind velocity estimation, i.e., the center of gravity and median estimators.

The mean (center of gravity) Doppler shift estimator, operating on a power spectral density (treated as a probability distribution function (PDF) of Doppler shifts), is

$\mu_{f}=\int f P_{r}(f) \mathrm{d} f / \int P_{r}(f) \mathrm{d} f$, 
where $\mu_{f}$ is the mean Doppler shift.

It can be easily shown that

$\int f P_{r}(f) \mathrm{d} f=2 \int f \Im\left[P_{i_{1} i_{\mathrm{Q}}}(f)\right]^{-} \mathrm{d} f$,

where $\Im\left[P_{i_{I} i_{Q}}(f)\right]^{-}$is the one-sided spectrum (see Abari et al., 2014). At first glance the center of gravity estimator should be able to operate on the cross-spectral approach. However, to estimate the center of gravity, the spectrum needs to be normalized, hence the normalization factor in the denominator of Eq. (11). Replacing $P_{r}(f)$ in Eq. (11) with $\Im\left[P_{i_{I} i_{Q}}(f)\right]^{-}$associated with the spectrum in Fig. $3 \mathrm{~d}$ results in $\Delta f$ which deviates from the true center of gravity estimate, i.e., $\left(\alpha^{2}-\beta^{2}\right) \Delta f /\left(\alpha^{2}+\beta^{2}\right)$.

The median estimator of the Doppler shifts is defined by

$$
\int_{-\infty}^{\tilde{f}} P(f) \mathrm{d} f=\frac{1}{2} \int_{-\infty}^{+\infty} P(f) \mathrm{d} f,
$$

where $\tilde{f}$ is the median frequency. It is easy to show that the median estimator for the average velocity retrieval fails to provide an accurate estimate when operating on $\Im\left[P_{i_{I} i_{Q}}(f)\right]^{-}$. As a result, the auto-spectrum of the signal, $P_{r}(f)$, needs to be utilized. The median estimator turns out to exhibit a lower variance (Angelou et al., 2012a), when compared to the center of gravity estimator. Once the median (or mean) value of Doppler shifts is estimated, it is easy to find the corresponding median wind speed by

$\widetilde{v}=\frac{1}{2} \lambda \tilde{f}$

Using the auto-spectrum in Eqs. (11) and (13) requires the dominant background noise to be removed (a rather signalprocessing-intensive procedure that can introduce an additional estimation error).

Following the above discussion, the cross-spectral approach cannot be reliably used when estimating either the mean or median value of the vertical wind component since there is a possibility for spectral cancellation across the zero frequency. The chances for spectral cancellation are even higher when measurements are carried out in turbulent flows and large sampling volumes. As shown in Eq. (5), the sampling volume increases quadratically as a function of distance from the transceiver antenna. Thus, more precautions should be taken when measurements are carried out for long ranges.

On the other hand, the cross-spectral approach is a very effective way for mean/median Doppler shift estimation in the event of Doppler spectra being confined to either side of the zero frequency. Hence, a combination of cross-spectral and auto-spectral approach can be employed for an efficient estimation of mean wind velocity in ICDLs. For instance, a real-time automated algorithm can primarily benefit from a cross-spectral approach to estimate the Doppler shifts. If

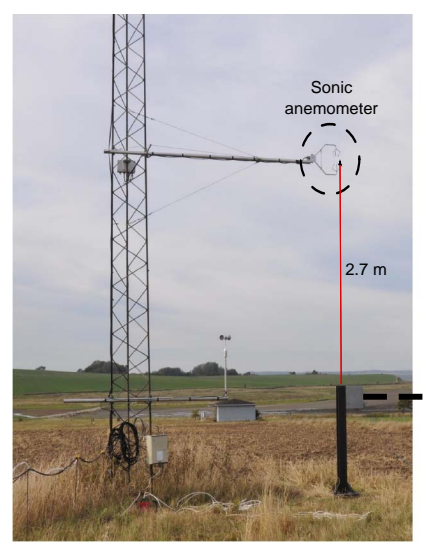

(a)

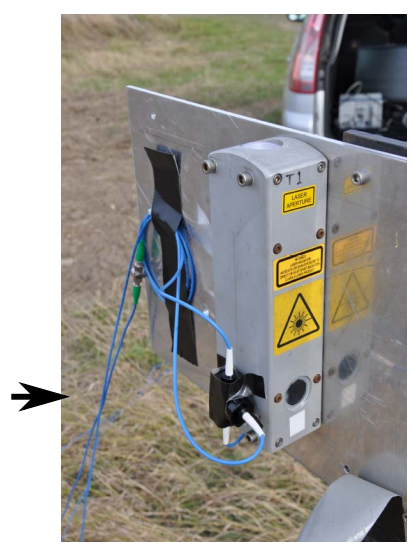

(b)
Figure 4. Field campaign at Ris $\varnothing$ campus of the Technical University of Denmark. (a) shows the position of the ICDL's output antenna with respect to the sonic anemometer. (b) is an expanded view of the mounting plate for the antenna, viewed from the backside of the plate seen in (a).

the estimated shift is inside a predefined confidence interval (e.g., $\pm 1 \mathrm{~m} \mathrm{~s}^{-1}$ ), the auto-spectral approach can be revoked to estimate the mean value of the Doppler shift.

In this paper, we have simply relied on the auto-spectral approach for the median Doppler shift estimation. This is justified by the fact that in this particular campaign we have purposefully performed the measurements for the vertical wind velocity component only. As we will see in Sect. 3, the results illustrate a significant improvement over the measurements performed by a HCDL.

\section{Measurement results}

Two separate and independent measurement campaigns were carried out to verify the results from the deployed $\mathrm{CW}$ CDLs against a sonic anemometer. In the first measurement campaign, carried out at the Ris $\varnothing$ campus of the Technical University of Denmark (October-November 2013), three HCDLs and one 3-D CSAT sonic anemometer (Cambell scientific) were utilized. The HCDLs were carefully positioned around the mast shown in Fig. 4a and focused on the measurement center of the sonic anemometer, which for this experiment was located around $6 \mathrm{~m}$ from the ground. The three wind lidars were tilted and measured at an angle of approximately $35^{\circ}$. The FWHM of the measurement volume was $90 \mathrm{~mm}$, which is comparable to the path length of the sonic anemometer $(115 \mathrm{~mm})$. The main purpose of this experiment at the time was to investigate the possibility of calibrating the sonic anemometer using the wind lidar. As mentioned before, only a subset of data representing wind measurements close to zero velocity, taken from only one HCDL, are used for comparison purposes in this paper. 


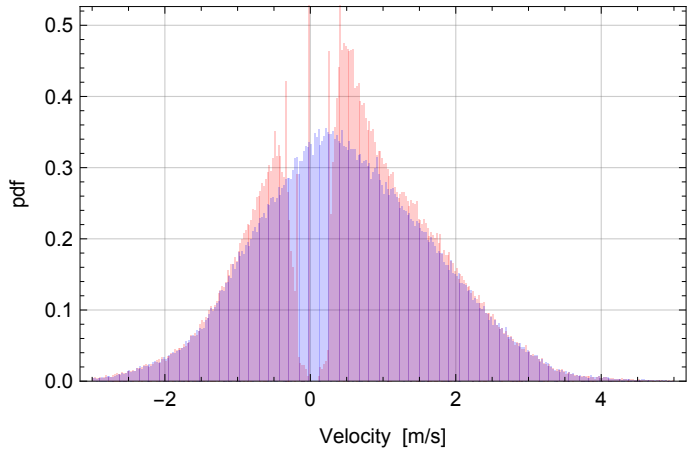

(a) Vicinity of zero velocity

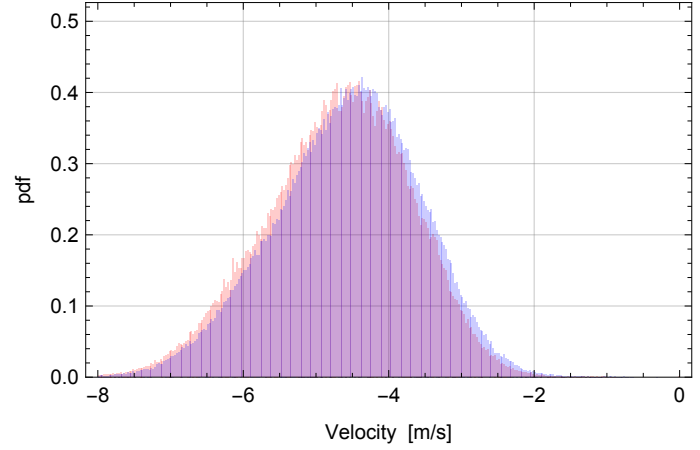

(b) Away from zero velocity

Figure 5. The PDF of estimated median velocities; in both figures, blue and red represent the measurements performed by the sonic anemometer and heterodyne CW CDL (HCDL), respectively. Please observe the gap in the PDF of velocities associated with the HCDL in (a). The overshoots (when compared to the blue PDF) correspond to the accumulation of the estimated velocities associated with the frequencies away from zero as well as the inaccurately estimated velocities associated with the frequencies inside the gap.

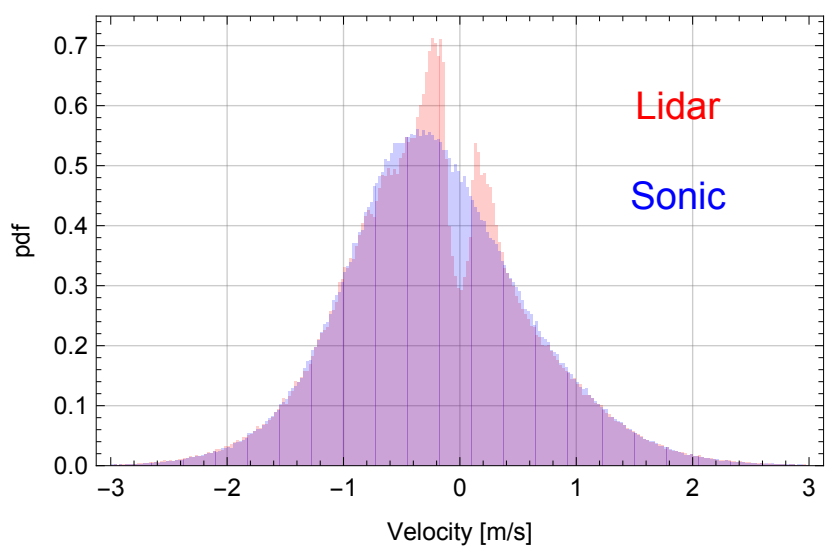

Figure 6. The PDF of the estimated median velocities close to zero, measured by the sonic anemometer and ICDL. Blue and red represent the sonic anemometer and lidar measurements, respectively. As we can infer from the gap in this figure, the ICDL also suffers from an estimation inaccuracy around zero. This can be attributed to spurious effects (such as DC offset, $1 / f$ noise, filtering, etc.) around the zero frequency. The noise behavior, however, is significantly improved when compared to the HCDL results presented in Fig. (5).

In a later measurement campaign, carried out in January 2014, we made use of a prototype ICDL elaborated in Abari et al. (2014). The parameters for the system are listed in Table 1 . To measure the vertical component of the wind, where observations of near-zero velocities are maximized, the beam at the output of the telescope was aligned vertically and the beam was focused at the measurement center of the sonic anemometer. Figure 4 shows the field deployment of the instrument for this specific campaign. Due to the direction of wind during both measurement campaigns, the effect of mast shadowing was minimal.
Figure $5 \mathrm{a}$ and $\mathrm{b}$ illustrate the PDF of the measured velocities for the measurement campaign carried out by the HCDL. Figure $5 \mathrm{~b}$ is an example associated with PDF of velocities away from the IF (zero Doppler shift) while Fig. 5a illustrates the PDF of velocities around the IF offset, i.e., zero radial velocity. As it can be seen from Fig. 5a and $b$ the performance of the lidar, compared against the sonic anemometer, is consistent across the displayed velocity range. ${ }^{3}$ However, the measured close-to-zero velocities are either impossible to estimate or significantly biased, when compared to the sonic anemometer. This is mainly due to the presence of spurious effects around the IF offset.

Figure 6 illustrates the PDF of the velocities measured by the ICDL, acquired during the latest field campaign. It is obvious that, when compared to the HCDL, the estimated mean velocities around zero are more consistent with the measurements performed by the sonic anemometer. This is mainly the direct consequence of using passive components for radial sign detection, elaborated in Abari et al. (2014), as opposed to the AOM (an active component), introducing additional spurious effects. Moreover, the need for notch filters, bandpass filters with a very narrow frequency band, for attenuating the strong IF offset is eliminated. From experience, the analog notch filters are costly, difficult to design, and often suffer from non-symmetric response. They also suffer from environmental effects such as temperature dependency. The image-reject receiver, though, benefits from a high-pass fil-

\footnotetext{
${ }^{3}$ In Fig. $5 \mathrm{~b}$ a shift of approximately $0.1 \mathrm{~m} \mathrm{~s}^{-1}$ can be seen between the sonic anemometer and the lidar velocity PDFs. The cause of this shift is presently unknown to us, but could be either due to flow distortion in the sonic anemometer or a slightly wrong rotation of the three-dimensional sonic velocity data. This difference is not the subject of the present contribution, but it will be pursued and addressed in a future paper.
} 
Table 1. Measurement campaign system parameters. $p_{t}, \mathrm{BW}$, and $f_{s}$ represent the optical output power, detection bandwidth, and sampling frequency, respectively. Furthermore, $N$ and $M$ represent the number of discrete Fourier transform (DFT) points and spectral averaging, respectively. Periodograms (Hayes, 1996) were used for the estimation of spectra as elaborated in Abari et al. (2014).

\begin{tabular}{ccccccccc}
\hline$F[\mathrm{~m}]$ & $D[\mathrm{~cm}]$ & FWHM $[\mathrm{mm}]$ & $\lambda[\mathrm{nm}]$ & $p_{t}[\mathrm{~W}]$ & $\mathrm{BW}[\mathrm{MHz}]$ & $f_{s}[\mathrm{MHz}]$ & $N$ & $M$ \\
\hline 2.7 & 2.2 & 140 & 1565 & 0.95 & 50 & 120 & 512 & 3900 \\
\hline
\end{tabular}

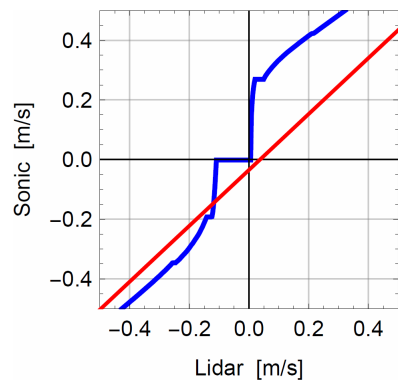

(a) Heterodyne CW CDL (HCDL)

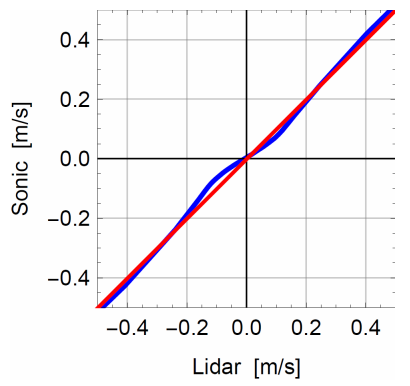

(b) Image-reject CW CDL (ICDL)

Figure 7. The estimated median velocities sorted in ascending order and stacked against the sonic anemometer (blue). The red line is a linear fit to the blue curve which extends to several $\mathrm{m} \mathrm{s}^{-1}$ in both directions. For an ideal lidar (and sonic anemometer) the blue curve would be a one-to-one line.

ter for removing the DC offset, which is more robust and has better frequency response characteristics.

Figure $7 \mathrm{a}$ and $\mathrm{b}$ show the estimated median velocities sorted in ascending order. The velocity range has been selected to be in the vicinity of the zero frequency shift. The estimated mean wind velocities, associated with the measurement volume, show a one-to-one correspondence between the sonic anemometer and lidar. Wind speed values in Fig. 7a and b are associated with data in Figs. 5a and 6, respectively. In these figures, the red curve is a linear fit to the measured data. An ideal one-to-one correspondence between the lidar and sonic anemometer should result in a straight line with a slope of one, passing through the center. For the campaign associated with the HCDL, Fig. 7a, a significant deviation from the reference instrument is observed (as expected). The deviations for the ICDL, Fig. 7b, are far less pronounced and consistently follow the sonic anemometer, except in a very narrow range around zero velocity.

\section{Conclusions}

The presented results in this paper verify the relevant performance improvement claims in Abari et al. (2014), where a prototype all-fiber CW CDL benefiting from an imagereject opto-electronic front-end was described. The comparison of the results from the presented system and a sonic anemometer clearly indicates a significant improvement in Doppler shift estimation over the AOM-based heterodyne receiver with IF sampling, especially for Doppler shifts close to zero. By discussing some special events, where the Doppler spectrum has energy both at negative and positive frequencies, we have shown that the auto-spectral approach, as opposed to the cross-spectral approach originally suggested in Abari et al. (2014), provides a more reliable estimation of the Doppler shifts. As a result, a hybrid approach to spectral estimation is desired where the algorithm primarily employs the cross-spectral approach but switches to an auto-spectral approach (with noise whitening) when small Doppler shifts of the order of $\pm 1 \mathrm{~m} \mathrm{~s}^{-1}$ are detected. 


\section{Appendix A: Abbreviations}

$\begin{array}{ll}\text { AOM } & \text { acousto-optic modulator } \\ \text { BW } & \text { bandwidth } \\ \text { CW } & \text { continuous wave } \\ \text { CDL } & \text { coherent Doppler lidar } \\ \text { DC } & \text { direct current } \\ \text { FWHM } & \text { full width at half maximum } \\ \text { HCDL } & \text { heterodyne coherent Doppler lidar } \\ \text { I } & \text { in-phase } \\ \text { ICDL } & \text { image-reject coherent Doppler lidar } \\ \text { IF } & \text { intermediate frequency } \\ \text { LO } & \text { local oscillator } \\ \text { PDF } & \text { probability distribution function } \\ \text { Q } & \text { quadrature-phase }\end{array}$


Acknowledgements. The authors would like to thank Xinzhao Chu from University of Colorado in Boulder and Mikael Sjöholm, Nikolas Angelou, and Karen Enevoldsen from the Technical University of Denmark for their invaluable help. This project is mainly funded by the WindScanner project from the Danish Strategic Research Council (DSF), Danish Agency for Science, Technology and Innovation; Research Infrastructure 2009; Grant No. 2136-08-0022. Additional funds from DSF grant no. 09-067216 (the DTU "Flow Center") are also appreciated. Ingeborg and Leo Dannin Grant for Scientific Research funded the NI computer used in this work.

Edited by: G. Ehret

\section{References}

Abari, C. F., Pedersen, A. T., and Mann, J.: An all-fiber imagereject homodyne coherent Doppler wind lidar, Opt. Express, 22, 25880-25894, 2014.

Angelou, N., Abari, C. F., Mann, J., Mikkelsen, T., and Sjöholm, M.: Challenges in noise removal from Doppler spectra acquired by a continuous-wave lidar, in: Proc. of the 26th Int. Laser Radar Conf., Porto Heli, Greece, 25-29 June, 2012, s5P01, 2012a.

Angelou, N., Mann, J., Sjöholm, M., and Courtney, M.: Direct measurement of the spectral transfer function of a laser based anemometer, Rev. Sci. Instrum., 83, 033111, doi:10.1063/1.3697728, 2012b.

Bingöl, F., Mann, J., and Larsen, G. C.: Lidar measurements of wake dynamics Part I: one dimensional scanning, Wind Energy, 13, 51-61, 2010.

Grund, C. J., Banta, R. M., George, J. L., Howell, J. N., Post, M. J., Richter, R. A., and Weickmann, A. M.: High-resolution Doppler lidar for boundary layer and cloud research, J. Atmos. Ocean. Tech., 18, 376-393, 2001.

Hall, F., Huffaker, R., Hardesty, R., Jackson, M., Lawrence, T., Post, M., Richter, R., and Weber, B.: Wind measurement accuracy of the NOAA pulsed infrared Doppler lidar, Appl. Optics, 23, 2503-2506, 1984.

Hayes, M. H.: Statistical Digital Signal Processing and Modeling, John Wiley \& Sons, New York, USA, 391-424, 1996.

Horst, T. W. and Oncley, S. P.: Corrections to inertial-range power spectra measured by CSAT3 and Solent sonic anemometers, 1. Path-averaging errors, Bound.-Lay. Meteorol., 119, 375-395, 2006.

Karlsson, C. J., Olsson, F. Å. A., Letalick, D., and Harris, M.: All-fiber multifunction continuous-wave coherent laser radar at $1.55 \mu \mathrm{m}$ for range, speed, vibration, and wind measurements, Appl. Optics, 39, 3716-3726, 2000.

Kylia: Single polarization $90^{\circ}$ optical hybrid $\mathrm{COH} 24$, available at: http://www.kylia.com/, last access: 23 September, 2015.
Mayor, S. and Eloranta, E. W.: Two-dimensional vector wind fields from volume imaging lidar data, J. Appl. Meteorol., 40, 13311346, 2001.

Menzies, R. T. and Hardesty, R. M.: Coherent Doppler lidar for measurements of wind fields, P. IEEE, 77, 449-462, 1989.

Mikkelsen, T., Mann, J., Courtney, M., and Sjöholm, M.: Lidarbased research and innovation at DTU wind energy - a review, J. Phys. Conf. Ser., 524, 012007, doi:10.1088/17426596/524/1/012007, 2014.

Moslehi, B.: Analysis of optical phase noise in fiber-optic systems employing a laser source with arbitrary coherence time, J. Lightwave Technol., 4, 1334-1351, 1986.

Pedersen, A. T., Abari, C. F., Mann, J., and Mikkelsen, T.: Theoretical and experimental signal-to-noise ratio assessment in new direction sensing continuous-wave Doppler lidar, J. Phys.: Conf. Ser., 524, 012004, doi:10.1088/1742-6596/524/1/012004, 2014.

Pichugina, Y. L., Banta, R. M., Brewer, W. A., Sandberg, S. P., and Hardesty, R. M.: Doppler lidar-based wind-profile measurement system for offshore wind-energy and other marine boundary layer applications, J. Appl. Meteorol. Clim., 51, 327-349, 2012.

Razavi, B.: Design considerations for direct-conversion receivers, IEEE T. Circuits-II, 44, 428-435, 1997.

Sathe, A. and Mann, J.: A review of turbulence measurements using ground-based wind lidars, Atmos. Meas. Tech., 6, 3147-3167, doi:10.5194/amt-6-3147-2013, 2013.

Schlipf, D., Cheng, P. W., and Mann, J.: Model of the correlation between lidar systems and wind turbines for lidar-assisted control, J. Atmos. Ocean. Tech., 30, 2233-2240, 2013.

Schwiesow, R. and Cupp, R.: Offset local oscillator for cw laser Doppler anemometry, Appl. Optics, 20, 579-582, 1981.

Shimizu, K., Horiguchi, T., Koyamada, Y., and Kurashima, T.: Coherent self-heterodyne Brillouin OTDR for measurement of Brillouin frequency shift distribution in optical fibers, J. Lightwave Technol., 12, 730-736, 1994.

Smith, D. A., Harris, M., Coffey, A. S., Mikkelsen, T., Jørgensen, H. E., Mann, J., and Danielian, R.: Wind lidar evaluation at the Danish wind test site in Høvsøre, Wind Energy, 9, 87-93, 2006.

Sonnenschein, C. M. and Horrigan, F. A.: Signal-to-noise relationships for coaxial systems that heterodyne backscatter from the atmosphere, Appl. Optics, 10, 1600-1604, 1971.

Urey, H.: Spot size, depth-of-focus, and diffraction ring intensity formulas for truncated Gaussian beams, Appl. Optics, 43, 620625, 2004.

Wang, Z., Liu, Z., Liu, L., Wu, S., Liu, B., Li, Z., and Chu, X.: Iodine-filter-based mobile Doppler lidar to make continuous and full-azimuth-scanned wind measurements: data acquisition and analysis system, data retrieval methods, and error analysis, Appl. Optics, 49, 6960-6978, 2010. 